\title{
Needle Stick Injuries among Healthcare Waste Handlers in a Tertiary Care Hospital of Delhi
}

\author{
Amrita Singh ${ }^{1}$, Surabhi Sethi ${ }^{2}$, Anita Verma ${ }^{3}$, Kriti Gangwar ${ }^{4}$, \\ Deepti Dabar ${ }^{5}$, Kundan Singh Rathore $^{6}$
}

\begin{abstract}
Introduction: World Health Organization (WHO) reports in the World Health Report 2002 that of the 35 million HCWs, 2 million suffer percutaneous exposure to infectious diseases each year. Healthcare waste handlers who are responsible for handling and collection of healthcare wastes are vulnerable to the hazards of occupational exposure to biomedical waste (BMW) as a consequence of improper disposal practices of the waste generators.
\end{abstract}

Objective: The study was conducted to determine the prevalence of Needle stick injury (NSI) among the study subjects, and to study the knowledge and practice regarding Needle stick injury (NSI) among the study subjects.

Methodology: A Hospital-based, cross-sectional study was conducted among healthcare waste handlers in a tertiary care hospital of Delhi. Of 390 healthcare waste handlers, 199 Bio-medical waste handlers were chosen on the basis of convenience according to their work shift.

Results: Sixty two (31.1\%) workers had knowledge that NSI during BMW handling can cause hepatitis and 127 (63.8\%) had knowledge that it can lead to HIV infection among them. The prevalence of NSI came to be $14.6 \%$ within the past six months.

Conclusion: A gap between knowledge and practice regarding action after NSI was found; therefore, measures to increase awareness are definitely required.

Keywords: Healthcare workers, Occupational injury, Occupational infection, waste handlers

\section{Introduction}

Needle stick injury (NSI) or percutaneous exposure incident is the penetration of skin by a needle or other sharp object, which was in contact with blood, tissue, or other body fluid before the exposure. ${ }^{1}$ Globally, NSIs are the most common source of occupational exposure and the primary cause of blood-borne infections of healthcare workers (HCWs). ${ }^{2}$ World Health Organization (WHO) reports in the World Health Report 2002 that of the 35 million HCWs, 2 million suffer percutaneous exposure to infectious diseases each year.

\footnotetext{
${ }^{1,6} \mathrm{MD},{ }^{2}$ Senior Resident, ${ }^{3}$ Professor, ${ }^{4}$ Graduate Student, Community Medicine, Vardhmann Mahavir Medical College and Safdarjung Hospital.

${ }^{5} \mathrm{MD}$ Community Medicine, Assistant Professor, Department of Community \& Family Medicine AlIMS, Bhopal, India.

Correspondence: Dr. Amrita Singh, Community Medicine, Vardhmann Mahavir Medical College and Safdarjung Hospital.

E-mail Id: amritasinghdr@gmail.com
}

Orcid Id: http://orcid.org/0000-0002-5747-4712

How to cite this article: Singh A, Sethi S, Verma A et al. Needle Stick Injuries among Healthcare Waste Handlers in a Tertiary Care Hospital of Delhi. Epidem Int 2017; 2(2): 14-17.

Digital Object Identifier (DOI): https://doi.org/10.24321/2455.7048.201706

ISSN: $2455-7048$ 
Almost $37.6 \%$ of Hepatitis B, 39\% of Hepatitis C and $4.4 \%$ of HIV/AIDS in HCWs globally are due to NSI, and the risk of transmission of infection from an infected patient to the HCW following a NSI are: Hepatitis B 3$10 \%$, Hepatitis C $3 \%$, and HIV $0.3 \%$. The two most common causes of NSIs are recapping of needles, and the unsafe collection and disposal of sharps waste. ${ }^{3}$ Many studies have been conducted among healthcare workers responsible for generating waste like doctors, nurses, laboratory technicians, but there are only a few studies which focus on Healthcare waste handlers responsible for handling and collection of healthcare wastes. This group of waste handlers is vulnerable to the hazards of occupational exposure to biomedical waste (BMW) as a consequence of improper disposal practices of the waste generators.

Hence the present study was conducted among healthcare waste handlers who were involved in collection, storage and disposal of BMW. The objectives of the study were: to determine the prevalence of NSI among the Bio-medical waste handlers and to study the knowledge and practice regarding NSI among the study subjects.

\section{Methodology}

A hospital-based, cross-sectional study was conducted among healthcare waste handlers in a tertiary care hospital of Delhi. Bio-medical waste handlers were defined as those hospital workers responsible for collection and/or transport of BMW generated in the hospital. ${ }^{4}$ A total of 390 workers formed our sample frame. Of 390 healthcare waste handlers, study subjects were chosen on the basis of convenience according to their work shift. Out of 390 waste handlers, 199 formed our study sample.

A semi-structured, interviewer-administered questionnaire was used to collect data. The questionnaire included questions regarding socio-demographic characteristics, knowledge and awareness of diseases transmitted due to NSI, vaccination and knowledge and practice regarding management of NSI. A sixmonth period prevalence of NSI was taken. Data collection was done from August to September 2015. Data thus collected was entered in MS Excel and analyzed for quantitative data. Mean, median, range, and qualitative data was expressed as frequency and proportions.

\section{Results}

Out of 199 participants, 162 (81\%) were male and 37 (18.5\%) were female. Among them, 163 (81.9\%) were married and 36 (18.1\%) were unmarried. The age of the participants ranged from 19 to 55 years, with the mean age of $30.6 \pm 8.05$ years.

Table1.Distribution of Study Participants according to Sociodemographic Profile

\begin{tabular}{|c|c|c|}
\hline \multicolumn{2}{|c|}{ Number (N=199) } & $\%$ \\
\hline Sociodemographic Profile & 7 & 3.5 \\
\hline$<20$ & 92 & 46.5 \\
\hline $20-30$ & 70 & 35.0 \\
\hline $30-40$ & 30 & 15.0 \\
\hline$>40$ & Religion \\
\hline Hindu & 191 & 96.0 \\
\hline Muslim & 6 & 3.0 \\
\hline Christian & 2 & 1.0 \\
\hline Illiterate & Educational status & 16.6 \\
\hline Primary & 33 & 14.1 \\
\hline Middle & 28 & 35.2 \\
\hline High & 70 & 26.1 \\
\hline Sr. Secondary & 52 & 6.0 \\
\hline Graduation & 12 & 2.0 \\
\hline Total & 4 & 100 \\
\hline
\end{tabular}

The average work experience of the study participants was 4.50 years, ranging from 2.0 to 6.0 years. There were $142(71.3 \%)$ waste handlers who had preplacement training and $29(14.6 \%)$ who had health checkup before job recruitment. All the study subjects ( $n=199,100 \%$ ) knew that personal protective equipment should be used while working, of which two participants $(1.0 \%)$ were not using any precautions while 
handling healthcare waste. One hundred ninety-seven (99.0\%) wore gloves, 195 (98.0\%) wore masks, 66 (33.2\%) wore aprons, and 40 (20.1\%) wore shoe covers while handling yellow and blue/metallic containers carrying sharps. Sixty-two (31.1\%) workers knew that NSI during BMW handling can cause hepatitis and 127 (63.8\%) had knowledge that it can lead to HIV infection among them. Of 199 study subjects, 46 $(23 \%)$ were aware that Hepatitis B vaccination is recommended, while only 17 (8.5\%) had ever received hepatitis $B$ vaccine.

There were 29 (14.6\%) study subjects who had a history of NSI within the past six months.

Table 2.Distribution of Study Participants according to Knowledge and Practice about What to Do following a Needle Stick Injury

\begin{tabular}{|c|c|c|}
\hline & Number & $\%$ \\
\hline \multicolumn{2}{|c|}{ Knowledge regarding Management of NSI (N=199)* } \\
\hline Consult the Medical Officer & 185 & 93.0 \\
\hline Let the wound bleed & 98 & 49.2 \\
\hline Wash wound with water and soap & 59 & 29.6 \\
\hline Wash wound with water only & 72 & 36.2 \\
\hline Press the wound with mouth & 37 & 18.6 \\
\hline Do nothing, continue to work & 4 & 0.2 \\
\hline \multicolumn{2}{|c|}{ Action Taken after NSI (n=29)* } \\
\hline Consulted the Medical Officer & 24 & 82.8 \\
\hline Did not follow up & 5 & 17.2 \\
\hline
\end{tabular}

*Multiple responses present

\section{Discussion}

In the present study, the prevalence of NSI in the past 6 months among healthcare waste handlers was found to be $14.6 \%$. Sharma et al., in their study conducted in Agra, found 19\% prevalence among the waste handlers. ${ }^{5}$ Ismail et al., in their study carried out in Karnataka (2012), found that $67 \%$ (20) of class IV waste handlers out of 30 had NSI during past 1 year. ${ }^{6}$ It must be noted that their recall period was one year as compared to 6 months in our study. However, the proportion of waste handlers in their studies is much smaller, therefore a correct comparison cannot be drawn. Shivalli et al. conducted a study in Mangalore on healthcare waste handlers among 43 workers, of which $41.8 \%$ had experienced NSI. ${ }^{7}$ Jahnavi et al. in their study conducted in Karnataka (2014) found 48 (64.9\%) of 74 housekeeping personnel had an occurrence of NSI. Also significantly higher mean frequency of NSI among housekeeping staff as compared to other healthcare workers was observed. ${ }^{8}$ Similar findings have been observed in the study carried out on 60 nursing homes of Delhi, where workers were engaged in harmful practices such as pressing injured finger in mouth, doing nothing after injury. ${ }^{9}$ The wide variation in prevalence and practices of NSI may be due to different length of time periods considered in different studies. In the present study, difference was observed between knowledge and practice of vaccination for prevention of hepatitis B. It can be concluded that waste handlers in the present study had a lower pre valence of NSI as compared to other studies and had good knowledge of management of NSI; however, their practice in terms of NSI prevention was not appropriate to their theoretical knowledge of the same.

Sample size was small; however, it was larger as compared to other studies. Recall bias might be present.

\section{Conclusion and Recommendation}

A gap between knowledge and practice regarding action after NSI was found; therefore, measures to increase awareness are definitely required. These could be displaying IEC materials, name and number of the persons to be contacted in case of NSI, infographics about problems due to NSI, etc. Apart from IEC/BCC activities, vaccination against Tetanus and Hepatitis $B$ should be made compulsory. Personal protective gear like masks, gloves and shoes should be made readily available and asked to wear before work.

\section{Acknowledgments}

The contribution of Dr. Vishwanath Pratap Singh, Dr. Wezode Wezah, Dr. Nitin Bhushan, Dr. Shalini Rawat, Dr. Ajeet Kumar, Dr. Paonam Babyrose Devi, Dr. Sadun, and Dr. Jitender is highly appreciated for data collection. We are thankful to healthcare waste handlers for giving us their precious time and to Hospital administration for giving permission to conduct the study.

Conflict of Interest: Nil 


\section{References}

1. CDC. The National Surveillance System For Healthcare Workers (NaSH): Summary Report for Blood and Body Fluid Exposure Data Collected from Participating Healthcare Facilities (June 1995 through December 2007). US, CDC, 2011. Available from: http://www.cdc.gov/nhsn/PDFs/NaSH/ NaSH-Report-6-2011.pdf (last accessed on Sep 19, 2016).

2. Centers for Disease Control and Prevention. National Institute for Occupational Safety and Health (NIOSH) NIOSH Alert: Preventing Needlestick Injuries in Healthcare Settings, 1999. Publication No. 2000-108.

3. World health organization. Aide-memoire for a strategy to protect health workers from infection with bloodborne viruses. Geneva, Switzerland: WHO November 2003. Available from: http:// www.who.int/occupational_health/activities/1am _hcw.pdf (last accessed on Dec 17, 2016).

4. Government of India. Policy on Hospital Waste Management Safdarjung Hospital 2010.
5. Sharma S, Gupta A, Arora A. Knowledge, attitude and practices on needle-stick and sharps injuries in tertiary care cardiac hospital: A survey. Indian J Med Sci 2010; 64: 396-401.

6. Ismail IM, Kulkarni AG, Kamble SV et al. Knowledge, attitude and practice about bio-medical waste management among personnel of a tertiary healthcare institute in Dakshina Kannada, Karnataka. Al Ameen J Med Sci 2013; 6: 376-80.

7. Shivalli S, Sowmyashree H. Occupational exposure to infection: A study on healthcare waste handlers of a tertiary care hospital in south India. $J$ Assoc Physicians India 2015; (63): 23-8.

8. Jahnavi R, Manjunath $M$, Mahendra BJ et al. Needle stick injury among healthcare workers in a government teaching hospital, Mandya. Int J Sci Stud 2014; 2(7): 103-06.

9. Kishore J, Agarwal R, Kohli $C$ et al. Status of biomedical waste management in nursing homes of Delhi, India. Journal of Clinical and Diagnostic Research 2014; 8(3): 56-58.

$$
\begin{aligned}
& \text { Date of Submission: } 22^{\text {nd }} \text { Jun. } 2017 \\
& \text { Date of Acceptance: } 23^{\text {rd }} \text { Jun. } 2017
\end{aligned}
$$

